University of Nebraska - Lincoln

DigitalCommons@University of Nebraska - Lincoln

9-26-2005

\title{
Metal hybridization and electronic structure of Tris(8-hydroxyquinolato)aluminum (Alq3)
}

\author{
Anthony N. Caruso \\ North Dakota State University, carusoan@umkc.edu \\ D. L. Schulz \\ North Dakota State University \\ Peter A. Dowben \\ University of Nebraska-Lincoln, pdowben@unl.edu
}

Follow this and additional works at: https://digitalcommons.unl.edu/physicsdowben

Part of the Physics Commons

Caruso, Anthony N.; Schulz, D. L.; and Dowben, Peter A., "Metal hybridization and electronic structure of Tris(8-hydroxyquinolato)aluminum (Alq3)" (2005). Peter Dowben Publications. 215.

https://digitalcommons.unl.edu/physicsdowben/215

This Article is brought to you for free and open access by the Research Papers in Physics and Astronomy at DigitalCommons@University of Nebraska - Lincoln. It has been accepted for inclusion in Peter Dowben Publications by an authorized administrator of DigitalCommons@University of Nebraska - Lincoln. 
Published in Chemical Physics Letters 413:4-6 (September 26, 2005), pp. 321-325; doi 10.1016/j.cplett.2005.07.098

Copyright @ 2005 Elsevier B.V. Used by permission. http://www.sciencedirect.com/science/journal/00092614

Submitted April 18, 2005; revised July 22, 2005; published online August 19, 2005.

\title{
Metal hybridization and electronic structure of Tris(8-hydroxyquinolato)aluminum $\left(\mathrm{Alq}_{3}\right)$
}

\author{
A. N. Caruso, ${ }^{*}$ D. L. Schulz, ${ }^{*}$ and P. A. Dowben ${ }^{\dagger}$ \\ * Center for Nanoscale Science and Engineering, North Dakota State University, \\ 1805 Research Park Drive, Fargo, ND 58102, USA \\ † Department of Physics and Astronomy and the Center for Materials Research and Analysis, \\ University of Nebraska-Lincoln, Lincoln, NE 68588-0111, USA \\ Corresponding author - A. N. Caruso, anthony.caruso@ndsu.edu
}

\begin{abstract}
The metal-organic complex Tris(8-hydroxyquinolato)aluminum $\left(\mathrm{Alq}_{3}\right)$ has been studied by energy and light polarization dependent photoemission. Resonant photoemission was used to identify the molecular orbitals involved in metal chelation. When adsorbed on cobalt and gold surfaces, marked differences in the $\mathrm{Alq}_{3}$ metal-to-ligand bonds were observed. The results indicate intramolecular aluminum-to-ligand bonding through the oxygen heteroatom when $\mathrm{Alq}_{3}$ is adsorbed on gold, but through the nitrogen heteroatom when on cobalt. These results indicate that substrate interfacial complex formation plays an important role in the $\mathrm{Alq}_{3}$ molecular configuration and intramolecular bonding.
\end{abstract}

\section{Introduction}

The metal-organic complex (OMC) Tris(8-hydroxyq uinolato)aluminum $\left(\mathrm{Alq}_{3}\right)$ is a widely utilized component in organic electroluminescent devices [1] and has recently shown promise as a thin non-magnetic layer in a giant magnetoresistive device [2]. Recent studies have suggested that charge transfer $[3,4]$ effects at the $\mathrm{Alq}_{3} /$ metal interface, as well as interface states $[5,6]$ and possibly metal induced gap states [5, 6] play an important role in charge injection and device performance. The alignment of the molecular orbitals with respect to the Fermi level and the electronic structure of a metal-organic/metal interface are expected to have a profound effect on charge injection in molecular electronics. These aspects of electronic structure are, in turn, influenced by the molecular interactions with the metal substrate. In the case of $\mathrm{Alq}_{3}$, there have been a number of studies which indicate that the band offset and charge polarity are key to device performance [3, $4]$, but the molecular configuration $[3,6]$ and pertur- bations to the Al-hydroxyquinolate bonds may have a more profound effect.

In this study, resonant photoemission is used to identify the molecular orbitals (MOs) associated with the complexed aluminum metal center and show that these molecular orbitals are strongly perturbed by the choice of metal substrate. The substrates were chosen to understand the metal-organic/metal interface formed in the magnetoresistive device (cobalt) compared to the more standard metal electrode (gold). The effect of molecular orbital perturbation is important to device performance, as already the quantum efficiency and color of luminescence in light emitting diodes composed from $\mathrm{Alq}_{3}$ has been shown to be dependent on the stereoisomer and phase of composition [7]. The two isomers of $\mathrm{Alq}_{3}$ are the facial and meridinal with $\mathrm{C}_{3}$ and $\mathrm{C}_{1}$ point group symmetries, respectively, shown in the inset of Figure 1. Other molecular configurations may exist as well, as "distortions" to the facial and meridinal structures of $\mathrm{Alq}_{3}$ in the "relaxed" geometries, as might occur in isolation. 


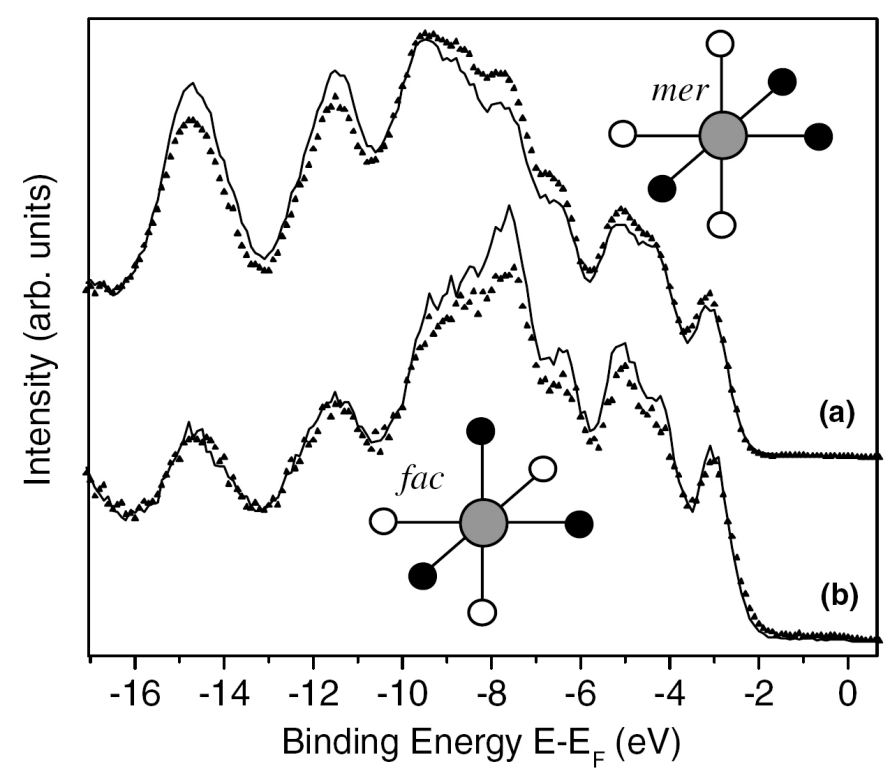

Figure 1. Polarization dependent photoemission at (a) $32 \mathrm{eV}$ and (b) $72.8 \mathrm{eV}$ photon energy of $\mathrm{Alq}_{3}$ adsorbed on $\mathrm{Au}(111)$. $\mathrm{p}$ polarization is represented by a solid line whereas $\mathrm{s}+\mathrm{p}$ polarization is the scatter plot $(\boldsymbol{\Delta})$. Inset are the octahedral metal coordination spheres for the meridinal and facial isomers of $\mathrm{Alq}_{3}$ with nitrogen and oxygen atoms as black and white circles, centered about an aluminum in gray.

\section{Experiment}

$\mathrm{Alq}_{3}$ was studied by angle resolved and polarization dependent ultraviolet photoemission spectroscopy under ultra high vacuum $\left(3 \times 10^{-10}\right.$ Torr $)$. The commercially purchased $\mathrm{Alq}_{3}$ powder (Aldrich) was purified before loading into an evacuated reservoir. The $\mathrm{Alq}_{3}$, adsorbed by vapor from sublimed powder, was admitted to the UHV chamber through a leak valve for adsorption on the UHV prepared metal thin film substrates.

The photoemission measurements, as described in detail elsewhere [8], were carried out at the Center for Advanced Microstructures and Devices synchrotron radiation facility in Baton Rouge, Louisiana with synchrotron light, monochromated by a $3 \mathrm{~m}$ torodial grating monochromator. $\mathrm{Alq}_{3}$ adsorptions were completed on cooled ( 100 K) epitaxial $\mathrm{Au} / \mathrm{Si}(111)$ and polycrystalline $\mathrm{Co} / \mathrm{Au} / \mathrm{Si}(111)$ substrates. The thicknesses of the $\mathrm{Alq}_{3}$ molecular layers, reported herein, are $14 \AA$. The gold coatings exhibited a (111) orientation by X-ray diffraction and both gold and cobalt coatings exceeded $100 \mathrm{~nm}$. All binding energies reported herein are referenced to the Fermi level as calibrated by tantalum foil in intimate contact with the sample surface with all photoelectrons collected normal to the substrate surface $\left(\mathrm{k}_{\|}\right.$ $=0$ or $\bar{\Gamma}$ ). Polarization dependent photoemission was ac- complished by varying the incident angle of the linearly polarized synchrotron light with the following provisions: $\mathrm{s}+\mathrm{p}$ polarization is given by $45^{\circ}$ incidence with respect to surface normal; and, $\mathrm{p}$ polarization is given by $70^{\circ}$ incidence, to yield the vector potential A more normal than parallel to the surface. The polarization dependence can be coupled to the photoemission selection rules under the local point group of adsorbed $\mathrm{Alq}_{3}$ to yield symmetry specific molecular orbital representations as a function of binding energy; the details of selection rule formalism are laid out elsewhere [9]. Energy dependent photoemission was employed to determine the bandwidth of molecular orbitals normal to the interface (for a crystalline overlayer thickness which did not exceed the mean free path of the substrate and its overlayer). The reciprocal space position normal to the interface $k_{\perp}$ is given as a function of incident photon energy by Eq. (1), where $h v$ denotes the incident photon energy, $E_{\mathrm{b}}$ the binding energy, $\theta$ is the emission angle $\left(0^{\circ}\right.$ in this work), the work function is given by $\varphi$ and $U$ represents the inner potential.

$$
k_{\perp}=\sqrt{\frac{2 m}{\hbar^{2}}\left\{\left(h v-E_{\mathrm{b}}-\varphi\right) \cos ^{2} \theta+U\right\}} .
$$

Resonant photoemission measurements were undertaken by comparing photoemission spectra collected with $h v=32$ and $72.8 \mathrm{eV}$ to be clearly off and on the Al $2 \mathrm{p}_{1 / 2} \rightarrow 3$ s (core to bound) absorption thresholds. The resonant photoemission, at $72.8 \mathrm{eV}$, is a coreto-bound excitation in which the incident photon excites an electron from the $\mathrm{Al} 2 \mathrm{p}$ core to an unoccupied state just above $E_{\mathrm{F}}$ that largely includes $\mathrm{Al}, \mathrm{O}$ and $\mathrm{N}$ weight. The bound electron decays, providing a resonant effect with the direct photoelectron emission process, and is localized to molecular orbitals with weight in the vicinity of the $\mathrm{Al} 2 \mathrm{p}$ core hole due to the strong Coulombic interaction.

\subsection{Aluminum to quinolinate ligand bonding for $\mathrm{Alq}_{3}$ at the substrate metal interface}

$\mathrm{Alq}_{3}$ does preserve some quinolinate to $\mathrm{Al}$ metal center bond symmetry. Figure 1 shows the light polarization dependent photoemission at incident photon energies of 32 and $72.8 \mathrm{eV}$ for $\mathrm{Alq}_{3}$ adsorbed on $\mathrm{Au}\left(\begin{array}{lll}1 & 1 & 1\end{array}\right)$ from vapor. The light polarization dependence, of the photoemission spectra, reveals that the $\mathrm{Alq}_{3}$ molecule, adsorbed on gold, does preserve some symmetry [9], particularly in the vicinity of the Al metal center. Although the light polarization dependence is different at different photon energies, the polarization dependence is significant for the photoemission feature at $-7.8 \mathrm{eV}$ binding energy. As this feature is enhanced at the $\mathrm{Al} 2 \mathrm{p}$ threshold (Figure 1), we can assign this feature to molecular orbitals that contain $\mathrm{Al}$ weight. 
With the $\mathrm{Alq}_{3}$ adopting a $\mathrm{C}_{3}$ point group symmetry, the irreducible representations that can be observed in photoemission are $a$ and $e$, where $a$ represents the symmetry axis of rotation $\left(120^{\circ}\right.$ through trisection of the octahedral oxygen and nitrogen) whereas $e$ signifies those molecular orbitals which have symmetries directions $\left(x, y, x z, y z, x^{2}-y^{2}\right)$ orthogonal to $a\left(z, x^{2}+y^{2}\right.$, $z^{2}$ ). The orthogonal symmetries of the facial $\mathrm{Alq}_{3}$ can be reconciled with the light polarization dependent photoemission, in contrast to the $\mathrm{C}_{1}$ point group meridional $\mathrm{Alq}_{3}$ isomer (with little or no symmetry). This identification of the facial $\mathrm{Alq}_{3}$ isomer, at least in the vicinity of the $\mathrm{Al}$ metal center, is also favored by others [10], but not all $[11,12]$.

As noted above for Figure 1, the light polarization dependent photoemission intensity is reversed as the photoemission cross section changes with incident photon energy. At $72.8 \mathrm{eV}$ photon energy, where there is resonant enhancement of molecular orbitals with aluminum weight, there is also enhancement of the same molecular orbitals (at $-7.8 \mathrm{eV}$ binding energy) with $\mathrm{p}$ polarized light. The polarization and energy enhancement indicates that the molecular orbitals with aluminum weight form as a result of atomic contributions with $\mathrm{s}$ and $\mathrm{p}_{z}$ components rather than the $\mathrm{p}_{x}$ or $\mathrm{p}_{y^{*}}$. The assignment of the $a$ irreducible representation may also apply to intramolecular bonding within the quinolate ligand, but is not necessary to satisfy the observed behavior. Such a picture of aluminum bonding to the octahedral nitrogen and oxygen orbitals, from the point of view of symmetry, is consistent with that proposed by Curioni et al. [11].

The light polarization dependence of the photoemission (Figure 1) is indicative of a strong preferential bonding orientation of $\mathrm{Alq}_{3}$ to the gold substrate. While the molecular film need not be crystalline, the results are consistent with strong texture to the molecular thin film growth in the thin film limit, unlike that suggested elsewhere for thicker molecular films [12].

For depositions of $\mathrm{Alq}_{3}$, from the vapor, on cobalt (Figure 2a), the photoemission reveals very different molecular orbital photoemission intensities and small increases in the $\mathrm{Alq}_{3}$ molecular orbital binding energies when compared to the molecular orbital induced photoemission features for $\mathrm{Alq}_{3}$ adsorption on gold (Figure $2 b$ ). As shown in Figure 2a, there is an obvious enhancement in photoemission intensity for the four major molecular orbitals photoemission features at $-4.6,-7.8$, -9.7 and $-11.9 \mathrm{eV}$ binding energy at the $\mathrm{Al} 2 \mathrm{p}$ resonance (taken at 72.8 versus $32 \mathrm{eV}$ photon energy). By way of comparison, as shown in Figure $2 b$, the photoemission spectra taken at the $\mathrm{Al} 2 \mathrm{p}$ resonance (again 72.8 versus $32 \mathrm{eV}$ photon energy) for $\mathrm{Alq}_{3}$ on Au show strongly enhanced features at -7.8 and $-5.2 \mathrm{eV}$ binding energies. The resonant enhancements in the $\mathrm{Alq}_{3}$ photoemission spectra demonstrate that the molecular orbitals with

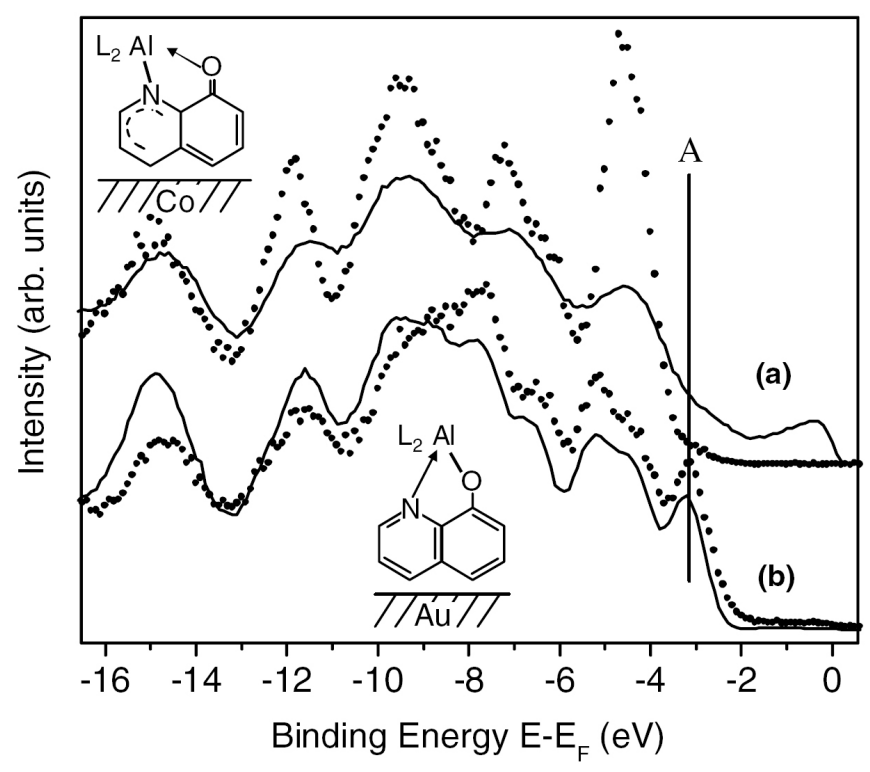

Figure 2. Photoemission of adsorbed $\mathrm{Alq}_{3}$ on (a) cobalt and on (b) gold at $32 \mathrm{eV}$ (solid line) and at $72.8 \mathrm{eV}(\bullet)$ incident photon energy. All spectra were taken by s + p polarization.

strong aluminum weight differ for $\mathrm{Alq}_{3}$ on cobalt than on gold.

If we compare the binding energies of the molecular orbitals, enhanced at the Al $2 p$ threshold in resonant photoemission, with the projected density of state calculations, provided by Curioni et al. [13] for each $\mathrm{Alq}_{3}$ component, we find that those molecular orbitals enhanced in Figure 2a are molecular orbitals with the nitrogen weight, whereas those photoemission features that are enhanced at the Al $2 p$ edge, in Figure $2 b$, are representative of molecular orbitals containing a strong oxygen projected density of states. These results indicate that for $\mathrm{Alq}_{3}$ adsorbed on cobalt, the $\mathrm{Al}$ metal center tends to bond the quinolinate ligands through the nitrogen whereas on gold, the $\mathrm{Alq}_{3}$ ligands bond to the $\mathrm{Al}$ more through the oxygen. This large difference in ligand-to-metal bonding suggests different molecular configurations are adopted by $\mathrm{Alq}_{3}$ at the different substrate surfaces.

Organometallic complex formation has been suggested for $\mathrm{Alq}_{3}$ adsorbed on magnesium and aluminum surfaces [6]. In the present study, interfacial $\mathrm{Alq}_{3}$ to metal substrate interaction induces intramolecular iminate ligand-to-metal bonds (i.e. Al-N) for $\mathrm{Alq}_{3}$ on cobalt and enolate (i.e., $\mathrm{Al}-\mathrm{O}$ ) bonds for $\mathrm{Alq}_{3}$ on gold. Furthermore, the fact that $\mathrm{Alq}_{3}$ adopts a very different molecular configuration at some interfaces does lend considerable weight to the interface model proposed by Rajagopal et al. [3,5] and others [6] and supports the premise that molecular configuration has a profound electronic effect at $\mathrm{Alq}_{3}$ interfaces. Nonetheless, $\mathrm{Al}$ to quinolate ligand molecular orbitals are identified for $\mathrm{Alq}_{3}$ adsorption on both gold and cobalt, in spite of the 
profound differences of the $\mathrm{Alq}_{3}$ electronic structure on the different substrates. Adsorption on both metals must be largely associative.

\subsection{The $\mathrm{Alq}_{3}$ density of states close to the fermi level}

An explanation of the effect of the substrate on the molecular orbital binding energies for condensed $\mathrm{Alq}_{3}$ are not easily addressed by a single factor such as work function. Prior studies have advocated two competing pictures for the $\mathrm{Alq}_{3}$ molecular orbitals relative position on metal surfaces. There are advocates of a model where the molecular orbitals are pinned to the Fermi level $[5,14]$ and others who have argued that charge transfer and substrate work function dominate $E_{\mathrm{F}}$ placement within the molecular HOMO-LUMO gap [4]. By compiling available photoemission spectra, a comparison of the position in binding energy of the highest occupied molecular orbital (HOMO) as shown in Figure 3, does not show a strong dependence upon substrate work function. Therefore, it seems more likely that the density of states near the Fermi level are dominated by impurities, decomposition or the different molecular phases now known to exist in the interfacial region, as demonstrated here and elsewhere $[3,5,6]$.

In comparing the spectral density between $\mathrm{Alq}_{3}$ adsorbed on gold versus cobalt, there is a much larger photoemission intensity at $-4.6 \mathrm{eV}$ binding energy and a very weak molecular orbital contribution to the photoemission spectra at $-3.1 \mathrm{eV}$ binding energy (labeled as " $A$ " in Figure 2). This absence of photoemission intensity for $\mathrm{Alq}_{3}$ on Co may be indirectly linked with a

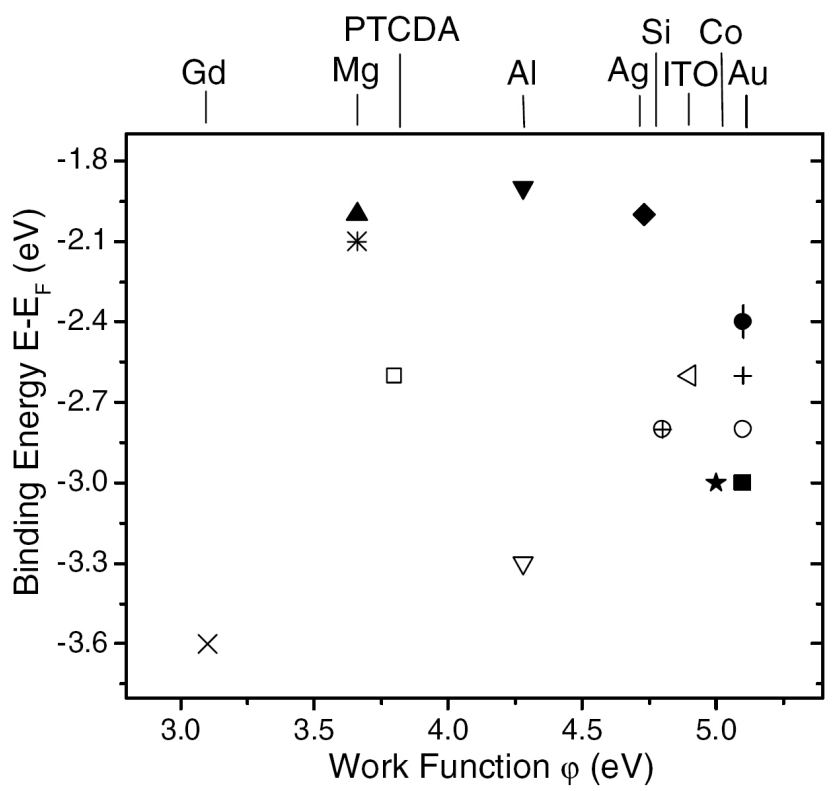

Figure 3. Comparison of the $\mathrm{Alq}_{3}$ highest occupied electronic state (state " $\mathrm{A}$ " in Figure 2) binding energy for various photoemission studies as a function of work function for the substrates used in the respective studies. The references to each symbol: $\star$, $\mathbf{\square - ~ t h i s ~ w o r k ; ~} \square[3] ; \times[4] ; \boldsymbol{\Delta}[5] ; *, \boldsymbol{\nabla}[6] ; \triangleleft, \bullet$ [14]; $\phi$ [16]; | [17]; ○ [18]; +, $\nabla$ [19]; $\diamond[20]$. previous study [2] where $\mathrm{Alq}_{3}$ film thicknesses under $100 \mathrm{~nm}$ deposited on cobalt, exhibited high resistances values in the range of $10^{4}-10^{5} \Omega$. That is, an absence of photoemission intensity for the HOMO indicates the absence of a density of states for the valence orbital and hence a greater insulating material.

The diminished $\mathrm{Alq}_{3}$ photoemission intensity at $-3.1 \mathrm{eV}$ binding energy for $\mathrm{Alq}_{3}$ when adsorbed on cobalt (vertical line " $A$ " in Figure 2a), does not appear to fit with a model of charge injection into $\mathrm{Alq}_{3}$ that relies upon the substrate work function [4]. This point is illustrated in Figure 3, where the highest occupied electronic state binding energies (derived from photoemission) for $\mathrm{Alq}_{3}$ on Co are compared with our results on $\mathrm{Au}$ and measurements of others for $\mathrm{Alq}_{3}$ on a variety of substrates. Again, there seems to be no obvious correlation between the highest occupied electronic state binding energy and substrate work function.

The presence of the photoemission peak " $\mathrm{A}$ " that was attributed to the highest occupied electronic state for $\mathrm{Alq}_{3}$ on gold, cannot be attributed to an interface state. Our reasoning is due to the small but finite dispersion of "A" in Figure 4, compiled from energy dependent photoemission. The presence of dispersion indicates that this peak is not localized to the interface, that is to say, the state does not preserve two dimensionality of state. Furthermore, peak " $\mathrm{A}$ " still exists for $\mathrm{Alq}_{3}$ films on many substrates (Figure 3), where the film thickness is much greater than the photoelectron mean free path. Hence the peak " $A$ " is due to the true $\mathrm{HOMO}$ and not an interface state as suggested in other studies $[6,15]$.

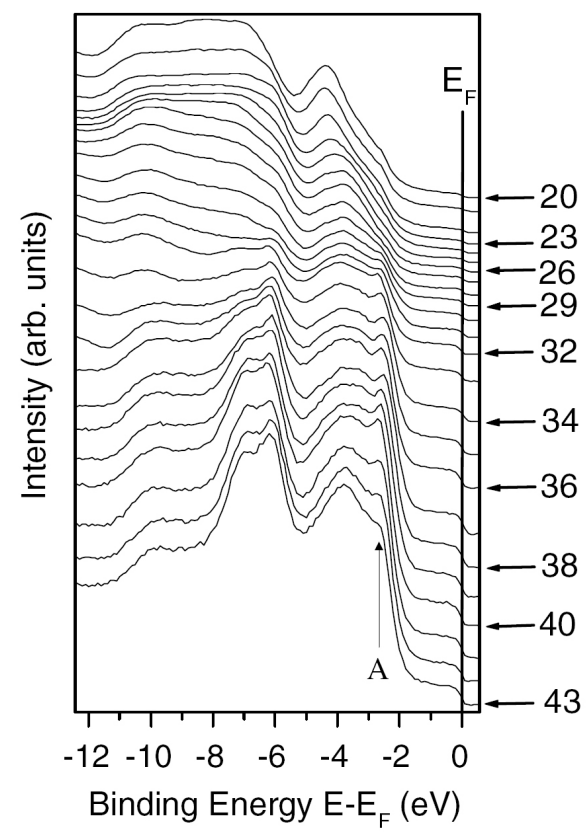

Figure 4. Energy dependent photoemission of $\mathrm{Alq}_{3}$ adsorbed on gold. The right side denotes the incident photon energies. Notice the photoemission cross section changes and dispersion directions as the photon energy is swept. All spectra were taken by p polarization. 
With the deposition of $\mathrm{Alq}_{3}$ on gold, the thin adlayers of $\mathrm{Alq}_{3}$ are periodic in the direction normal to the surface. As observed in Figure 4, the photoemission features due to the $\mathrm{Alq}_{3}$ highest occupied electronic state at $-2.7 \mathrm{eV}$ and at least two other $\mathrm{Alq}_{3}$ photoemission features at -6.1 and $-7.0 \mathrm{eV}$ binding energy $(h v=43 \mathrm{eV})$ exhibit little dispersion, while the photoemission features due to the $\mathrm{Alq}_{3}$ molecular orbitals at -3.7 and $-9.8 \mathrm{eV}$ binding energy $(h v=43 \mathrm{eV})$ exhibit significant photon energy dependence. These changing binding energies with photon energy occur over small values of $k_{\perp}$ consistent with the fact that $\mathrm{Alq}_{3}$ is a larger molecule compared to most molecular adsorbates with molecular orbital wave vector dependence.

\section{Conclusion}

We have investigated the occupied electronic structure of $\mathrm{Alq}_{3}$ adsorbed on epitaxial $\mathrm{Au} / \mathrm{Si}(111)$ and polycrystalline cobalt. The light polarization dependent photoemission indicates the presence of the facial isomer of $\mathrm{Alq}_{3}$. The suppression of highly lying occupied $\mathrm{Alq}_{3}$ states adsorbed on $\mathrm{Co}$ is consistent with the large resistance values [2] for $\mathrm{Alq}_{3}$ on Co. The relative binding energy positions of the highest occupied electronic state, as compared in Figure 3, cannot be easily explained by charge transfer or the work function of the metals; rather it is the interface conditions which ultimately dictate the binding energy positions. Issues pertaining to charge injection into the molecular film will undoubtedly be affected by both the deposition method, which dictates the physical structure of the molecular film, as well as the complications that arise from the substrate dependent hybridization.

Overall, the molecular orbital structure of $\mathrm{Alq}_{3}$ [16] is far from complete unless the influence of the substrate is considered. In this regard Kahn and coworkers [3, 6] are correct that the details of the interface matter. The results presented here indicate that interface states are partly the result of the changes in the configuration and geometry of the $\mathrm{Alq}_{3}$ molecules caused by substrate interaction that, in turn, induce intramolecular iminate or enolate bonding rather than by the substrate to molecule interaction alone [6]. Arguments involving band bending are difficult to invoke in a system where the molecular configuration and intramolecular bonding configuration changes so dramatically at an interface. The intermolecular interactions, by comparison, are likely quite weak so that a rigid band model seems more likely to be applicable (except at the interface). States that have been interpreted as interface states are shown by photon energy dependent photoemission to be attributable to $\mathrm{Alq}_{3}$ alone and disperse normal to the surface for a crystalline $\mathrm{Alq}_{3}$ thin film grown on $\mathrm{Au}$. In spite of the agreement of the results here with Curioni et al. [13], the role of $\mathrm{Al}$ in the molecular orbitals is quite significant and the Al-ligand bond can contribute significantly to the highest occupied molecular orbitals; a result not directly suggest by Curioni and coworkers $[11,13]$. We may summarize by noting that the aluminum seems to play a very small role in intermolecular bonding but a large, although indirect role in intramolecular bonding induced by the metal substrate.

\section{Acknowledgments}

This work was supported by the National Science Foundation through Grant CHE-0415421 and the NSF “QSPINS” MRSEC (DMR-0213808), the Defense Microelectronics Activity (DMEA) under agreement CMEA90-02-2-0218 and the NSF through ND EPSCoR Grant EPS-0447679. The authors also wish to thank the Center for Advanced Microstructures and Devices, which is funded by the State of Louisiana. The authors also wish to thank Yaroslav Losovyj and Rob Sailer for the help with experimental setup.

\section{References}

1. C.W. Tang and S.A. VanSlyke, Appl. Phys. Lett. 51 (1987), p. 913.

2. Z.H. Xiong, Di Wu, Z. Valy Vardeny and Jing Shi, Nature $\mathbf{4 2 7}$ (2004), p. 821.

3. A. Rajagopal, C.I. Wu and A. Kahn, J. Appl. Phys. 83 (1998), p. 2649.

4. S.C. Kim, S.N. Kwon, M.-W. Choi, C.N. Whang, K. Jeong, S.H. Lee, J.-G. Lee and S. Kim, Appl. Phys. Lett. 79 (2001), p. 3726.

5. A. Rajagopal and A. Kahn, J. Appl. Phys. 84 (1998), p. 355.

6. C. Shen, A. Kahn and J. Schwartz, J. Appl. Phys. 89 (2001), p. 449.

7. M. Braun, J. Gmeiner, M. Tzolov, M. Cölle, O. Wendland, F.D. Meyer, W. Milius, H. Hillebrecht, J.U. Schütz and W. Brütting, J. Chem. Phys. 114 (2001), p. 9625.

8. P.A. Dowben, D. LaGraffe and M. Onellion, J. Phys.: Condens. Matter. 1 (1989), p. 6571.

9. E.W. Plummer and W. Eberhardt, Adv. Chem. Phys. 49 (1982), p. 533.

10. M. Cölle, R.E. Dinnebier and W. Brütting, Chem. Commun. (2002), p. 2908.

11. A. Curioni, M. Boero and W. Andreoni, Chem. Phys. Lett. 294 (1998), p. 263.

12. M. Brinkmann, G. Gadret, M. Muccini, C. Taliani, N. Masciocchi and A. Sironi, J. Am. Chem. Soc. 122 (2000), p. 5147.

13. A. Curioni, W. Andreoni, R. Treusch, F.J. Himpsel, E. Haskal, P. Seidler, C. Heske, S. Kakar and L.J. Terminello, Appl. Phys. Lett. 72 (1998), p. 1575.

14. S.T. Lee, X.Y. Hou, M.G. Mason and C.W. Tang, Appl. Phys. Lett. 72 (1998), p. 1593.

15. C. Shen and A. Kahn, Org. Electron. 2 (2001), pp. 89-95.

16. L.S. Liao, X.H. Sun, L.F. Cheng, N.B. Wong, C.S. Lee and S.T. Lee, Chem. Phys. Lett. 333 (2001), p. 212.

17. K. Sugiyama, D. Yoshimura, T. Miyamae, T. Miyazaki, H. Ishii, Y. Ouchi and K. Seki, J. Appl. Phys. 83 (1998), p. 4928.

18. I.G. Hill, A. Kahn, J. Cornil, D.A. dos Santos and J.L. Brédas, Chem. Phys. Lett. 317 (2000), p. 444.

19. S.N. Kwon, S.C. Kim, Y. Kim, J.-G. Lee, S. Kim and K. Jeong, Appl. Phys. Lett. 79 (2001), p. 4595.

20. A. Schmidt, M.L. Anderson and N.R. Armstrong, J. Appl. Phys. 78 (1995), p. 5619. 